The future of information under the CBD, Nagoya Protocol,

Plant Treaty and PIP Framework

Charles Lawson, ${ }^{1}$ Fran Humphries ${ }^{1}$ and Michelle Rourke ${ }^{1,2}$

${ }^{1}$ Australian Centre for Intellectual Property in Agriculture

Griffith Law School

Griffith University

Gold Coast Queensland 4222

AUSTRALIA

${ }^{2}$ Australian Defence Force Malaria and Infectious Disease Institute

Gallipoli Barracks

Enoggera Queensland 4051

AUSTRALIA

$\mathrm{T}-+61(07) 55527249$

E-c.lawson@griffith.edu.au

michelle.rourke@griffithuni.edu.au

frances.humphries@griffith.edu.au 


\title{
The future of information under the CBD, Nagoya Protocol, \\ Plant Treaty and PIP Framework
}

\begin{abstract}
The United Nations' Convention of Biological Diversity (and the Nagoya Protocol on Access to Genetic Resources and the Fair and Equitable Sharing of Benefits Arising from their Utilization), the Food and Agriculture Organisation of the United Nations' International Treaty for Plant Genetic Resources for Food and Agriculture and the World Health Organisation of the United Nations' (WHO) Pandemic Influenza Preparedness Framework all set out schemes for access and benefit sharing (ABS) some biological materials. This article addresses the apparent conflict between the general obligations in these agreements to disclose and exchange information and dealing with information as a resource derivative within the $A B S$ transaction. This latter dealing is a closed domain for information under the ABS schemes where information is a resource derivative that is part of the ABS transaction. Treating information as a resource derivative within the ABS transaction is likely to impose unnecessary and inefficient burdens on ABS transactions. After reviewing the recent developments, the article postulates a risk framework for valuing information as a part of the ABS transaction, or alternatively, a charge, tax or levy to externalize the costs so that information remains available to be disclosed and exchanged promoting more and better science and research.
\end{abstract}

Keywords: ABS, information, CBD, Nagoya Protocol, Plant Treaty, PIP Framework

\section{Introduction}

The United Nations' Convention of Biological Diversity (CBD, 1992), the Nagoya Protocol on Access to Genetic Resources and the Fair and Equitable Sharing of Benefits Arising from their Utilization to the Convention on Biological Diversity (Nagoya Protocol, 2010) as a supplementary agreement to the CBD, the Food and Agriculture Organisation of the United Nations' International Treaty for Plant Genetic Resources for Food and Agriculture (Plant Treaty, 2001) and the World Health Organisation of the United Nations' (WHO) Pandemic Influenza Preparedness Framework (PIP Framework, 2011) all set out schemes for access and benefit sharing some biological materials (CBD, Art 15; Nagoya Protocol, Art 6; Plant Treaty, 
Art 12; PIP Framework, Art 5.1; see Lawson, 2012, pp. 125-239). Each of these schemes provides for an agreement between the resource holder and the party seeking access, being an individually negotiated agreement under the CBD (Art 15) and the Nagoya Protocol (Art 6), or standard material transfer agreements (SMTAs) under the Plant Treaty (Arts 12.4 and 15.1; IT/GB-1/06/Report, 2006, [12] (Resolution 2/2006) and Appendix G) and PIP Framework (Art 5.4 and Annexes 1 and 2). Under the CBD and the Nagoya Protocol these biological materials are "genetic resources" comprising "any material of plant, animal, microbial or other origin containing functional units of heredity" (CBD, Art 2; Nagoya Protocol, Art 2; UNEP/CBD/WG$A B S / 7 / 2,2008,[18]$ and Annex ([3]). In practice, however, this definition has a very flexible meaning and those implementing the CBD and Nagoya Protocol generally apply the term broadly to include most biological materials and derivatives (UNEP/CBD/WG-ABS/9/INF/1, 2010; see also UNEP/CBD/COP/3/20, 1996, [35]-[37]). [1] The Plant Treaty applies to biological materials that are "any genetic material of plant origin of actual or potential value for food and agriculture" where "genetic material" means "any material of plant origin, including reproductive and vegetative propagating material, containing functional units of heredity" (Art 2). The Plant Treaty then narrowly addresses only materials that are available for facilitated access in the Multilateral System (Arts 10 and 11), and these are primarily a list of agriculturally important "Annex 1" materials that have been identified as publicly available (Art 11). The PIP Framework only applies to a narrow range of pandemic influenza viruses comprising "human clinical specimens, virus isolates of wild type human H5N1 and other influenza viruses with human pandemic potential; and modified viruses prepared from H5N1 and/or other influenza viruses with human pandemic potential developed by [the] WHO" (Art 4.1).

The CBD, Nagoya Protocol, Plant Treaty and the PIP Framework also include general information obligations independent of the ABS contracting obligations (CBD, Arts 16.1, 17.1, 17.2, 18.3; Nagoya Protocol, Art 14; Plant Treaty, Art 17; PIP Framework, Art 5.1.3). These general information obligations are intended to promote the disclosure and exchange of information. They are, however, different and contrast with the information treated as a derivative of the materials within the ABS transaction itself, that becomes a distinct commodity with a value that $A B S$ attempts to translate into definable benefits. The concern of this article is the conflict between information as an element of $A B S$ (so a resource 
derivative) and the general information obligations to make information broadly available. The following quote captures the concern in the context of sharing virus "genetic sequence data" (GSD) under the PIP Framework where information that falls within the general information obligations substitutes for the physical materials that are otherwise a part of the ABS transaction, in effect, eviscerating the benefit sharing within the ABS transaction:

GSD and physical materials are dealt with differently under the PIP Framework ... GSD is not included in the definition of [PIP Framework biological materials], and there is no mechanism (trigger) to operationalize the requirement for benefit sharing from GSD. Thus, there is a dissonance between the way the PIP Framework treats GSD and the reality in which it is used by scientists. This dissonance, if not resolved soon, could threaten the relevance of the PIP Framework since the sharing of GSD largely operates outside the virus sharing and benefit sharing rules of the PIP Framework (A70/17, 2017, Annex (p. 49)).

Each of the CBD, Nagoya Protocol, Plant Treaty and the PIP Framework governing bodies have started to address this problem in the context of "digital sequence information" under the CBD, Nagoya Protocol and Plant Treaty (UNEP/CBD/COP/DEC/14/20, 2018; UNEP/CBD/COP/13/25, 2016, [321] and Decision XIII/16 ([1]); IT/GB-7/17/Report, 2017, [43] and Appendix (Resolution 13/2017, [2])), and GSD under the PIP Framework (A70/17, 2017 Annex (pp. 25-26 and 48-54); see also A69/22 Add.1, 2016, Annex ([14]-[19]); A69/22 Add.2, 2016, Annex 2). The problem, however, is much broader and applies wherever the physical material is substitutable by information when there is sufficient knowledge about the physical materials that further knowledge derived from the physical material is not required for the particular application ("de-materialization" - IT/GB-5/13/Report, 2013, Appendix I.2). Put slightly differently, this problem arises whenever information can replace the physical materials and the information exchanged in its own right (IT/GB-5/13/4, [3]). For the purposes of this article, and reflecting the uncertainty about appropriate terminology (see UNEP/CBD/DSI/AHTEG/2018/1/3, 2018, [63]-[82]), we use the phrase "genetic information" as a collective to refer to information derived from the genetic resources, plant materials and viruses. The apparent solution at the CBD, Nagoya Protocol, Plant Treaty and the PIP Framework forums is to deal with genetic information as a resource derivative that is part of the $A B S$ transaction to address the evisceration of benefit sharing within the ABS transaction (see, for example, IT/GB-7/17/Report, 2017, [26] and Appendix (Resolution 2/2017, Annex 2, [13]-[14])). 
The purpose of this article is to review the current state of considering genetic information in the CBD, Nagoya Protocol, Plant Treaty and the PIP Framework fora and then to address the consequential problems of enclosing information with the physical materials when dealing with information as a resource derivative within the ABS transaction. The next part addresses whether the terminology "genetic resources" and the like is sufficiently broad to include information about the genetic resources (so a resource derivative) to show that the terminology is sufficiently flexible and that the consensus appears to be against any renegotiation of the terminology. The following parts reviews the developments in considering genetic information under each of the CBD, Nagoya Protocol, Plant Treaty and the PIP Framework as a resource derivative and then the arrangements in place to address the general information obligations to disclose and exchange information. The article then considers that a closed domain for information under the ABS schemes will directly conflict with the open domain required by the information sharing obligations, and that shifting the handling of information into the ABS domain is an enclosure of information beyond the existing ideals of confidential information and trade secrets. The contribution of this article is to postulate a risk framework (matrix) for valuing information as a part of the ABS transaction, or alternatively, a charge, tax or levy to externalize the costs so that information remains available to be disclosed and exchanged promoting more and better science and research.

\section{2. "Genetic resources"/plant "genetic materials"/pandemic influenza viruses and information}

The question is whether the term "genetic resources" in the CBD (Art 2), the Nagoya Protocol (Art 2), and similar terms in the Plant Treaty (Art 2: "plant genetic resources for food and agriculture") and PIP Framework (Art 4.1: "PIP biological materials"), are broad enough to include genetic information. That the term "genetic resources" probably refers to the physical genetic resources is apparent from the definitional text in the CBD. The CBD defined the terms "country of origin of genetic resources" as "the country which possesses those genetic resources in in situ conditions" where "in situ-conditions" means "conditions where genetic resources exist within ecosystems and natural habitats, and, in the case of domesticated or cultivated species, in the surroundings where they have developed their distinctive properties" (Art 2). Further, the terms "country providing genetic resources" is defined to 
mean "the country supplying genetic resources collected from in situ sources, including populations of both wild and domesticated species, or taken from ex situ sources, which may or may not have originated in that country" (Art 2). Defined in this context, "genetic resources" appears to be the physical materials that make up the biological materials that are the object of the exchange in the ABS transaction. There is, however, some flexibility in the terms.

The early discussions about the CBD accepted that "genetic resources" could extend to genetic material found in humans because some of the materials from humans was "material of animal origin containing functional units of heredity" and "could provide insight into the evolution of the human species as well as the nature of human susceptibility and resistance to diseases" making it material of "actual or potential value" (UNEP/CBD/COP/2/13, 1995, [64]). This was, however, cautioned against - "from the history of its negotiation, it is clear that the $[C B D]$ was not formulated with human genetic resources in mind" (UNEP/CBD/COP/2/13, 1995, [64]). Subsequent Conferences of the Parties confirmed that human genetic resources are not within the ambit of either the CBD (UNEP/CBD/COP/2/19, 1995, [106] and Annex II (Decision II/11, [2])) or the Nagoya Protocol (UNEP/CBD/COP/10/27, 2010, [103] and Annex (Decision $X / 1,[5])$ ) even though they might satisfy the textual definition. Further, the Contracting Parties also accepted that the "genetic resources" addressed by the CBD were only those accessed after 29 December 1993, with those collected before this date outside the realm of the CBD (e.g. UNEP/CBD/COP/4/27, 1998, [223]). While there is this guidance about the scope of "genetic resources" from the Conference of the Parties, Contracting Parties do have the freedom to interpret the term according to their interests, and they do. For example, Malaysia's Access to Biological Resources and Benefit Sharing Act 2017 (Mal) s 12(2)(f) specifically includes human genetic resources within its ABS scheme. These examples demonstrate that the term does have some imposed limits and is not strictly defined for the purposes of the CBD. Rather the term as used in the CBD and Nagoya Protocol appears to be narrower that the potential meaning of "genetic resources" although it does retain considerable flexibility.

In contrast, more recently the legal object of these physical genetic resources has been expanded beyond the original CBD textual definition. While the CBD apparently dealt with 
only "genetic resources" as "genetic material of actual or potential value" (CBD, Art 2; see also UNEP/CBD/COP/2/13, 1995, [51]-[53]), disquiet among Contracting Parties about the narrow reach of this definition to other biological materials and chemicals that might have value in ABS transaction has been countered by drafting domestic implementing laws that also include biochemicals and derivatives (Wynberg and Laird, 2009, p. 81). For example, Australia's ABS legislation uses the broad terminology of "biological resources" rather than genetic resources and then narrows the scope of their application with specific inclusions and exclusions (Environment Protection and Biodiversity Conservation Act 1999 (Cth) s 528; Biodiscovery Act 2004 (Qld) s 5 and Sch; Biological Resources Act 2006 (NT) ss 4 and 5). [2] This example demonstrates an application beyond the strict meaning and much broader than the term used in the CBD and Nagoya Protocol.

A study commissioned by the CBD Secretariat on the possible meanings of "genetic resources" confirmed that the meaning is potentially "dynamic and flexible" (UNEP/CBD/WG$A B S / 9 / I N F / 1,2010$, p. 34). The study concluded that with a narrow definition "the ABS system may not be able to capture the future potential value of genetic material, not least when it is used in or as a basis for synthetic biology or other new bio-economic technologies" (UNEP/CBD/WG-ABS/9/INF/1, 2010, p. 34). The later Nagoya Protocol appears to have embraced an expansion with its definitions of "utilization of genetic resources" and "derivative" (Art 2). Importantly, however, the Nagoya Protocol adopted additional definitions while retaining the CBD definitions (Art 2) rather than just expanding the existing meaning of the text "genetic resources". And during these negotiations it was apparent that a renegotiation of the definitions in the CBD was not considered practical (e.g. UNEP/CBD/WG-ABS/7/2, 2008, [18] and Annex ([6])). Intriguingly, the Nagoya Protocol's definition of "utilization of genetic resources" includes "to conduct research and development on the genetic and or biological composition of genetic resources" and this might be broadly interpreted so that it "could entail that the generation or analysis of genomic data qualifies as research on, hence utilization of, the genetic resource" (IT/GB7/SAC-1/16/BSP 10, 2016, p. 8 and the references therein).

Under the Plant Treaty the definition of "plant genetic resources for food and agriculture" refers to "genetic material" that in turn is defined as a physical material as "any material of 
plant origin, including reproductive and vegetative propagating material, containing functional units of heredity" (Art 2). The inclusion of "functional units of heredity" is consistent with the CBD (and Nagoya Protocol) and engages the same kinds of discussions about the likely meanings. Again, there does not seem to be any moves to renegotiate the definitions.

And under the PIP Framework "PIP biological materials" includes "human clinical specimens, virus isolates of wild type human $\mathrm{H} 5 \mathrm{~N} 1$ and other influenza viruses with human pandemic potential; and modified viruses prepared from H5N1 and/or other influenza viruses with human pandemic potential developed by WHO GISRS laboratories" and "RNA extracted from wild-type H5N1 and other human influenza viruses with human pandemic potential and cDNA that encompass the entire coding region of one or more viral genes" (Art 4.1). As confirmation that these current definitions only embody the physical materials the PIP Framework separately defines "genetic sequences" as "the order of nucleotides found in a molecule of DNA or RNA. They contain the genetic information that determines the biological characteristics of an organism or a virus" (Art 4.2). This suggests a physical embodiment of the nucleotides in the molecule of DNA or RNA and that the information in the genetic sequences is something different.

These analyses of the texts show that the definition of "genetic resources" is not clear and fixed and that there is some dynamism and flexibility in the term (UNEP/CBD/WG$A B S / 9 / I N F / 1,2010$, p. 34). At this stage it is not clear whether an expanded scope of the term "genetic resources" to include genetic information will be favoured even though some countries have already adopted expansive definitions that capture genetic information (see Bagley and Rai, 2014, p. 20). There are, however, credible suggestions that the current term is dated and ill-suited to the developments in science and technology that the ABS schemes were intended to address (egs. Muller, 2018, pp. 212-213; Laird and Wynberg, 2016, 192-193; Bagley and Rai, 2014, pp. 20-24; UNEP/CBD/WG-ABS/9/INF/1, 2010, p. 5; Schiele et al., 2015, p. 101; and so on). For example, in defining "genetic resources" to include "functional units of heredity" the latter terms are not defined by either the CBD or the Nagoya Protocol, and it is not clear whether a partial coding DNA sequence or a DNA sequence that regulates gene expression constitutes a "functional unit of heredity" (Bagley and Rai, 2014, p. 20). This would 
be a significant impediment to future $A B S$ arrangements because partial sequences and regulatory elements are likely to be significant uses of accessed materials. For our purposes, however, the significance of these analyses is that the scope of the CBD, Nagoya Protocol, Plant Treaty and the PIP Framework may not be sufficiently broad to capture genetic information, and as a consequence, the mandatory obligations for benefit sharing may be avoided. In practice and application, however, it is apparent that countries implementing the CBD (and Nagoya Protocol) have expanded the meaning of "genetic resources" to include a broad range of materials and this can be sufficient to capture genetic information if desired. This pragmatic approach is probably appropriate as the Nagoya Protocol negotiations confirmed that a renegotiation of the definitions in the CBD is not considered practical (e.g. UNEP/CBD/WG-ABS/7/2, 2008, [18] and Annex ([6])).

\section{Information in the ABS schemes}

Each of the CBD, Nagoya Protocol, Plant Treaty and the PIP Framework governing bodies has started to engage with the problem that genetic information might be used in ways that avoid the benefit sharing obligations of ABS. The quintessential example driving this engagement is accessing DNA sequence information about a genetic resource on a publicly accessible database of sequences and using that accessed DNA sequence information to derive some benefit. An example would be manufacturing and selling a vaccine developed using DNA sequence information without accessing the physical virus materials (e.g. Noyce et al., 2018). This part reviews the engagements of the CBD, Nagoya Protocol, Plant Treaty and the PIP Framework so far.

\subsection{CBD and Nagoya Protocol}

The Conference of the Parties to the CBD has considered "digital sequence information" as a cross-cutting issue of concern to achieving the objectives of the CBD (UNEP/CBD/COP/DEC/14/20, 2018; UNEP/CBD/COP/13/25, 2016, [321] and Decision XIII/16 (pp. 124-125); see also UNEP/CBD/COP/13/5, 2016, [113] and Recommendation XX/8 ([o] and Annex ([1(e)]), p. 75); UNEP/CBD/SYNBIO/AHTEG/2015/1/3, 2015, [52] ([m], p. 15)). The outcomes were to request the Secretariat to prepare a compilation and synthesis of the views, commission a fact-finding and scoping study, establish an Ad Hoc Technical Expert Group on Digital Sequence Information on Genetic Resources, and join this to similar 
initiatives under the Nagoya Protocol (UNEP/CBD/COP/13/25, 2016, [321] and Decision XIII/16 ([3], [4] and [6], p. 124); see also UNEP/CBD/NP/MOP/2/13, 2016, [84] and Decision $2 / 5$ ([4], p. 14)). These developments are presently underway and have been extended with outcomes to be reported at the next Conference of the Parties in 2020 (UNEP/CBD/COP/DEC/14/20, 2018, [12]). At this stage the fact-finding and scoping study has been completed (see UNEP/CBD/DSI/AHTEG/2018/1/3, 2018) and the Ad Hoc Technical Expert Group will be convened with five experts nominated by each of the five regions (UNEP/CBD/COP/DEC/14/20, 2018, [11]-[12]). The fact-finding study concluded that there are a range of challenges to realizing benefits from utilizing "digital sequence information" because of "the difficulties of monitoring and identifying contributors, users and the provenance of sequences; the problems of determining value; and the increasingly grey area between non-commercial and commercial research" (UNEP/CBD/DSI/AHTEG/2018/1/3, 2018, Annex ([50] and [252])). In addressing "digital sequence information", the Nagoya Protocol's Conference of the Parties essentially joined the processes under the CBD (UNEP/CBD/NP/MOP/2/13, 2016, [84] and Decision 2/5 ([4], p. 14) and Decision 2/14 (p. 38); UNEP/CBD/COP/13/25, 2016, [321] and Decision XIII/16 (pp. 124-125)).

\subsection{Plant Treaty}

The Commission on Genetic Resources for Food and Agriculture established a new work stream on "digital sequence information on [genetic resources for food and agriculture]" [3] to undertake an exploratory fact-finding scoping study to better understand the types and extent of uses of such information, its relevance for food security and nutrition, and the implications of its use "for the conservation and sustainable use of genetic resources for food and agriculture, including exchange, access and the fair and equitable sharing of the benefits arising from their use" (CGRFA-16/17/Report, 2017, [86]). The outcomes were to be shared with the CBD processes and reported back to the Commission on Genetic Resources for Food and Agriculture at its next meeting (CGRFA-16/17/Report, 2017, [87]-[91]). In parallel with this process, the Plant Treaty's Governing Body decided to consider the potential implications of "digital sequence information" [4] at its next meeting (IT/GB-7/17/Report, 2017, [43] and Appendix (Resolution 13/2017, [2])). In the meantime, the Plant Treaty Secretariat was to seek information about the types and extent of uses of such information and its relevance for food security and nutrition, for a future discussion about the implications of its use for the 
objectives of the Plant Treaty including the "exchange, access and the fair and equitable sharing of the benefits arising from their use" (IT/GB-7/17/Report, 2017, [43] and Appendix (Resolution 13/2017, [4] and [5])). In anticipation of the Plant Treaty's Governing Body considering "digital sequence information" a special event on genomics information was convened with a commissioned study (see IT/GB-7/17/Report, [43] and Appendix A.13 (Resolution 13/2017)). The commissioned study concluded that "even if a robust tracking system were possible, other factors including partial sequence combinations, and the fact that the same sequence may occur in multiple organisms, further challenge the $A B S$ principles" (Welch et al. 2017, p 15).

Like the Commission on Genetic Resources for Food and Agriculture, these outcomes from the Governing Body meetings were to be shared with the CBD and the Commission on Genetic Resources for Food and Agriculture processes and reported back to the Governing Body at its next meeting (IT/GB-7/17/Report, 2017, [43] and Appendix (Resolution 13/2017, [5]-[7])). The Governing Body also tasked the reconvened Scientific Advisory Committee on the Global Information System of Article 17 to consider the "scientific and technical issues of relevance to digital sequence information as far as it is generated from the use of [plant genetic resource for food and agriculture] and related to the implementation of [Global Information System]" and report back to the next meeting (IT/GB-7/17/Report, 2017, [43] and Appendix (Resolution 13/2017, [8] and [10])). The Scientific Advisory Committee at its next meeting considered that applying digital object identifiers (DOIs) to information could be a useful starting point and that developing quality standards for DOI relationships linking material and information might be useful (IT/SAC-GLIS-3/18/Report 2018, [19]-[21]).

\subsection{PIP Framework}

Unlike the other schemes the PIP Framework does envision dealing with "genetic sequences" (see PIP Framework, Art 5.2 and Annex 5 (WHO Collaborating Centres for Influenza, [4] and [4]; WHO H5 Reference Laboratories, [5] and [6])). These are defined as DNA and RNA molecules that "contain the genetic information that determines the biological characteristics of an organism or a virus" (Art 4.2). The PIP Framework expressly provides that "genetic sequence data" (GSD), relatedly termed "digital sequence information" under the CBD, Nagoya Protocol and Plant Treaty (see UNEP/CBD/COP/13/25, 2016, [321] and Decision 
XIII/16 ([1]); IT/GB-7/17/Report, 2017, [43] and Appendix (Resolution 13/2017, [2])), and "analyses arising from that data" should be "shared in a rapid, timely and systematic manner" within the WHO laboratories (Art 5.2.1), and submitted to GISAID [5] and Genbank [6] or similar databases (PIP Framework, Art 5.2 .2 and Annexes 4 ([9]) and 5 ([9])). Aware of the sensitivities of this issue, and particularly that viruses are tracking through the uses of the SMTA 2 outside the WHO laboratories and real time track through the Influenza Virus Traceability Mechanism into, within and out of the WHO system (Art 5.3) while no such requirement applies to GSD, the PIP Framework Advisory Group was tasked with finding resolution to "handling of genetic sequence data" (Art 5.2.4 and Annex 3).

The PIP Framework Advisory Group (A66/17, 2013, Annex 1 (pp. 23-24)) started work on the best process to handle the use of influenza virus GSD under the PIP Framework (A67/36, 2014, Annex 1 ([4]-[6])). To assist the Advisory Group a Technical Expert Working Group on Genetic Sequence Data (TEWG) was convened (see A67/36, 2014, Annex 1 ([5])) to provide "[a]n assessment of the scientific, technical, operational and intellectual property implications of using [influenza viruses with pandemic potential] GSD rather than physical materials for influenza research and vaccine production, including how the transfer of such data could be monitored" (PIP TEWG, 2014, p. 2). The TEWG usefully identified the possible uses of GSD: the direct uses of GSD where there is the use of a particular sequence to develop a product, such as "the production of 'synthetic' candidate vaccine viruses ... for vaccine development" (PIP TEWG, 2014, pp. 3-4); the use of bulk sequences where multiple gene or genome sequences are analyzed or used in bulk and no single virus can be considered critical (PIP TEWG, 2014, pp. 4-5); and indirect uses of GSD where the sequences assist in achieving some other goal, such as generating a protein to derive antibodies for therapy, diagnostics, and so on, epidemiologic studies for robust migration analysis of the virus, predicting vaccine efficacy based on viral evolution models founded on GSD, and so on (PIP TEWG, 2014, p. 5). Interestingly, for our purposes, the TEWG also considered whether it was "possible or feasible to monitor or trace the sharing of GSD"? (PIP TEWG, 2014, pp. 10-12). The TEWG identified sharing GSD informally or formally like a written document, through structured media like as databases, and through ad hoc methods like an email (PIP TEWG, 2014, p. 10). While each of these were possible, the PIP Framework also guides the release of GSD through GISAID and GenBank or similar databases (PIP Framework, Art 5.2.2 and Annexes 4 ([9]) and 5 ([9]); PIP 
TEWG, 2014, p. 10). These databases can be publicly-accessible unrestricted databases that allow anybody to submit sequences, download sequences and use sequences for any purpose (like GenBank), publicly-accessible restricted databases that are accessible to the public but require users to register and accept certain terms and conditions before they can access and use the database (like GISAID), and private or closed databases that restrict access to users that have been identified and accepted as users (PIP TEWG, 2014, p. 11). As the TEWG notes, however, GSD from materials accessed under the PIP Framework may be indistinguishable from GSD generated outside the PIP Framework and with no involvement of the PIP Framework participants (PIP TEWG, 2014, p. 11). This means that tracing GSD into products for benefit sharing involves more than just tracking GSD. To address this problem, the TEWG proposed: click-wrap agreements for sharing GSD derived from PIP Framework materials where possible, such as downloading from databases, with a voluntary system of notification for the purposes of the PIP Framework; monitoring products generated from GSD using the GSD as identifying data in scientific publications, patent applications, regulatory product approvals, and so on; and possible restrictions imposed through the SMTA 2 where materials are shared with entities outside the WHO such as pharmaceutical vaccine and antiviral manufacturers (PIP TEWG, 2014, pp. 11-12).

The PIP Framework Advisory Group then sought consultations with representatives of databases, industry and civil society and concluded that "[t]he objective of benefit sharing may be met by mechanisms related to monitoring products generated using influenza GSD, rather than by monitoring use of GSD and/or tracing GSD" (PIP FAG DG, 2014, [31c]). The Advisory Group further considered systems to monitor use of GSD in end-products in consultation with the WHO laboratories, databases and industry and other stakeholders (PIP FAG DG, 2014, [32]). The TEWG was again tasked with "defining the optimal characteristics of a GSD sharing system" consistent with the PIP Framework and the "fair and equitable access to benefits generated using [PIP Framework] GSD" (PIP FAG TWG, 2014, p. 3; see also PIP FAG TWG GSD, 2016, Annex 2 (p. 25)). After several meetings the TEWG submitted a final report (PIP FAG TWG GSD, 2016, Annex 2 (p. 25)), with the meetings notably addressing the concern that GSD submitted according to the PIP Framework should not imperil academic or scientific publication (PIP FAG TWG GSD, 2015, [18]-[24]). The final report only addressed GSD accessed from databases and identified two immediate forms of access as databases with registered 
user access or controlled access databases (so called "DRUA/CAD" or "public-access" databases like GISAID) and database without access agreement or open-access database (so called "DWRUA/OAD" or "public-domain" databases like GenBank) (PIP FAG TWG GSD, 2016, pp. 1 and 4). The options for the optimal characteristics of a GSD sharing system were identified as: (1) monitoring data users such as using electronic logs or self-reporting and possibly coupled with monitoring GSD in commercial products; and (2) monitoring GSD in commercial products (PIP FAG TWG GSD, 2016, p. 1). The TEWG considered that monitoring data users was really only possible through registered user access or controlled access databases (DRUA/CAD) (PIP FAG TWG GSD, 2016, p. 1).

The Advisory Group also asked the PIP Framework Secretariat to consider benefit sharing mechanisms for GSD including options for monitoring the uses of GSD (see GSD Monitoring, 2016, pp. 2-4). The Secretariat presented various possible options but did not address either how to operationalize the considered options or the responsible implementing entity (GSD Monitoring, 2016, p. 4). The options were framed in the context of upstream and downstream - "[u]pstream options should focus on informing entities and individuals accessing GSD of potential obligations and/or expectations under the PIP Framework" while "downstream options would involve implementing mechanisms to monitor use of Influenza Viruses with Human Pandemic Potential GSD (or IVPP GSD) to develop end-products, such as vaccines, antivirals and diagnostics" (GSD Monitoring, 2016). Among the upstream options was consideration that databases without access agreement or open-access databases ("DWRUA/OAD" or "public-domain" databases like GenBank) could notify users of potential third-party claims over the data either through statements typically found in user terms of use or identifying the PIP Framework GSD in the metadata of sequences provided to the databases (GSD Monitoring, 2016, pp. 5-6). Meanwhile user access or controlled access databases ("DRUA/CAD" or "public-access" databases like GISAID) could impose terms of use limiting the further distribution of GSD to only other users that had also agreed to the terms of use, include a notifying statement about expectation of users, include metadata requirements about the origin and use of data (like unique identifiers such as accession numbers), and share user activity on the databases with the WHO (GSD Monitoring, 2016, pp. 6-7). The downstream options considered were about methods of monitoring the use of GSD after it has been shared and used to research and develop end-products through unique 
identifiers (such as accession numbers) in publications, patent applications and regulatory approval files (GSD Monitoring, 2016, pp. 7-8). As the Review Group notes, however, users of GSD are not required to use or disclose unique identifiers and not all uses of GSD will be apparent in end products (such as GSD used to test products) (GSD Monitoring, 2016, p. 8). The downstream mechanisms to identify GSD uses that were considered were search engines to retrieve information from publicly available documents like scientific publications, published results, patents, research trial documentation and regulatory files (GSD Monitoring, 2016, pp. 10-15). The remaining downstream options considered was merely to seek voluntary disclosures and trace GSD uses through references to the physical materials like the names of viruses (GSD Monitoring, 2016, pp. 15-16).

In compliance with the PIP Framework mandate for a review (Art 7.4.2), a Review Group conducted a review of, among other things, the handling of GSD (A70/17, 2017, Annex (pp. 25-26 and 48-54); see also A69/22 Add.1, 2016, Annex ([14]-[19]); A69/22 Add.2, 2016, Annex 2). The Review Group proposed amending the PIP Framework to clarify that GSD was included as part of the PIP Framework materials (pandemic influenza viruses) so that they would be included in the PIP Framework tracking mechanism (Influenza Virus Traceability Mechanism into, within and out of the WHO system) (A70/17, 2017, Annex (pp. 53-54); PIP Framework, Art 5.3). They also proposed amendments to clarify that submission of GSD sequences should be to a "publicly accessible database" hence favoring user access or controlled access databases ("DRUA/CAD" databases like GISAID) that can impose terms of use limiting the further distribution of GSD to only other users that have also agreed to the terms of use, and perhaps accepting the intractable nature of tracking GSD that "there are diverse views on the optimal traceability and monitoring mechanism, [so] the Advisory Group should give consideration to broadening and deepening engagement with all stakeholders" (A70/17, 2017, Annex (pp. 53-54)).

The recent discussions about the PIP Framework at the WHO appear to have expanded the existing discussions with proposals for the World Health Assembly to consider both GSD and the broader effects of the Nagoya Protocol (see EB144/CONF./10, 2019). The next World Health Assembly may be expected to deliver more concrete proposals, although it is clear that the discussion of GSD and the work of the Advisory Group's outcomes will continue. 


\section{Information sharing obligations}

The analyses so far show that the terminology used to define the materials covered by the CBD, Nagoya Protocol, Plant Treaty and the PIP Framework have some flexibility, and that there can be a distinction between the physical materials and the information about those materials. As a matter of practice, however, Contracting Parties implementing the CBD and Nagoya Protocol have adopted definitions to suit their national circumstances (see, e.g., Environment Protection and Biodiversity Conservation Act 1999 (Cth) s 528; Biodiscovery Act 2004 (Qld) s 5 and Sch; Biological Resources Act 2006 (NT) ss 4 and 5), and in some instances have adopted definitions that embrace information as part of the ABS arrangements. For example, Malaysia's Access to Biological Resources and Benefit Sharing Act 2017 (Mal) s 4 provides "biological resource" includes "any information relating to" genetic resources, populations and biotic components. There also appears to be a pragmatic approach under each of the international schemes not to start renegotiating definitions (see UNEP/CBD/WG$A B S / 7 / 2,2008,[18]$ and Annex ([6]). The more problematic issue is the apparent conflict between any information derived from accessed physical materials as part of the ABS transaction and the general information obligations intended to promote the disclosure and exchange of information without any of the benefit sharing obligations. This part considers the information sharing without benefit sharing obligations under the CBD, Nagoya Protocol, Plant Treaty and the PIP Framework.

\subsection{CBD and Nagoya Protocol}

The CBD sets out a general obligation applying to all the CBD's objectives for the exchange of information about the "results of technical, scientific and socio-economic research", "training and surveying programmes", "specialized knowledge", "[I]ndigenous and traditional knowledge as such and in combination with the technologies ['that are relevant to the conservation and sustainable use of biological diversity or make use of genetic resources']" (Arts 16.1 and 17.1). There is also a Clearing House Mechanism (CHM) "to promote and facilitate technical and scientific cooperation" (Art 18.3). [7] The CHM is being realized through a decentralised collection of information hubs (databases and websites) and national government websites with very little formal regulation (see UNEP/CBD/COP/12/29, [149] and Decision XII/2, (pp. 12-18); UNEP/CBD/WGRI/5/3/Add.2). The CHM is essentially a pointer to 
other websites holding the information. The separate and centrally located Nagoya Protocol Access and Benefit Sharing Clearing House is also being developed as a part of the CBD's CHM, although it applies more narrowly to only the ABS arrangements and not the broader CBD ambit of $A B S$, information sharing and technology transfer (Art 14.1; UNEP/CBD/ICNP/3/6).

Both the CBD and Nagoya Protocol do not affect the rights and obligations under any existing international agreement "except where the exercise of those rights and obligations would cause a serious damage or threat to biological diversity" (CBD: Art 22.1; Nagoya Protocol: Art 4.1). Consistent with these obligations both the CBD and Nagoya Protocol also make express provision respecting that access and transfer of technology "shall be provided on terms which recognize and are consistent with the adequate and effective protection of intellectual property" (CBD: Art 16.2; Nagoya Protocol, Art 4.4). The effect of these provisions is to accept the existing measures regulating information under the World Trade Organisation's TradeRelated Aspects of Intellectual Property Rights (TRIPS) (TRIPS, 1994) in the form of confidential information, trade secrets and undisclosed information used in regulatory decision making about pharmaceutical and agricultural chemical products (Art 39; see Malbon et al., 2014, pp. 571-595).

\subsection{Plant Treaty}

The Plant Treaty and its SMTA operate together to regulate the exchanges of shared plant materials including various information obligations placed on Plant Treaty's Contracting Parties and the parties to the SMTA to make information available (Art 12.3(c); IT/GB1/06/Report, 2006, [12] (Resolution 2/2006) and Appendix G (Art 6.9)). Central to this arrangement is a Global Information System (GLIS) to allow the information about plant materials to be collected, stored and shared with all potential users of the Plant Treaty's materials (Art 17). The GLIS also functions as an early warning system to safeguard threatened materials (Art 17.2) and to assist the Commission on Genetic Resources for Food and Agriculture to periodically assess the state of the world's genetic resources (Art 17.3). The kinds of information in the GLIS includes "catalogues and inventories, information on technologies, results of technical, scientific and socio-economic research, including characterization, evaluation and utilization, regarding those plant genetic resources for food and agriculture" that is not confidential (Art 13.2(a)). Like the CBD's CHM and Nagoya 
Protocol's Access and Benefit Sharing Clearing House, the GLIS is being implemented as a decentralised network of databases and websites with the GLIS developing only as a pointer to these other databases (IT/GB-6/15/Report, 2015, [31] (Resolution 3/2015) and Appendix A.3, Annex 1).

Like the CBD and Nagoya Protocol, the Plant Treaty does not alter the rights and obligations of parties under other international agreements (Plant Treaty, Preamble). Further, the Plant Treaty's objectives are intended to be "closely linked" with the CBD (and Nagoya Protocol) (Art 1.2). Again, the effect of these provisions is to accept the existing measures regulating confidential information, trade secrets and undisclosed information used in regulatory decision making about pharmaceutical and agricultural chemical products under TRIPS (Art 39; see Malbon et al., 2014, pp. 571-595).

\subsection{PIP Framework}

Under the PIP Framework providers of human clinical specimens, influenza virus isolates, extracted RNA, cDNA, and influenza candidate vaccine viruses (see Arts 2.1(i) and 4.1) to WHO-coordinated network of laboratories (Art 5.1.1) authorise sharing "information as agreed in the Influenza Virus Traceability Mechanism" (Art 5.1.3(ii)). The Influenza Virus Traceability Mechanism is the computer system for tracking the transfers and movements of physical materials into, within and out of the WHO network of laboratories (Art 4.4). Where the physical materials are analyzed within the WHO network of laboratories the "[g]enetic sequence data and analyses arising from that data" is shared (Art 5.2.1). The WHOcoordinated network of laboratories is then required to submit genetic sequence data to GenBank and GISAID or similar database in a timely manner consistent with the SMTA (Art 5.2.1 and Annexes 1 ([5.1.1]), 4 ([9]) and 5([9])).

\section{Conclusions}

The purpose of this article was to review the current state of engaging genetic information under the CBD, Nagoya Protocol, Plant Treaty and the PIP Framework as a resource derivative and then to address the consequential problems of enclosing information within the ABS transaction. The analyses so far has shown that each of the CBD, Nagoya Protocol, Plant Treaty and the PIP Framework have been framed according to the ABS schemes applying to 
physical materials and their physical derivatives. The best practice example that framed the CBD negotiations illustrating this physicality was the 1992 agreement between the National Biodiversity Institute of Costa Rica (INBio) and the United States pharmaceutical company Merck (see Roberts, 1992; UNEP/CBD/COP/2/13, 1995, Annex I (p. 31)). According to this agreement Merck collected possible valuable chemicals for pharmaceutical and agricultural applications from Costa Rica's rainforests, and in exchange for access, agreed to pay INBio fixed fees and royalties and transferred technology including laboratory equipment and supplies, training, and so on (UNEP/CBD/COP/2/13, 1995, Annex I (p. 31)):

The deal exemplifies how governments of the South ... could be paid to provide companies with access to genetic resources ... [and] ... could serve as a model which governments of the South can emulate to generate income from their genetic resources (Svarstad, 1994, p. 54).

As the INBio example demonstrates, the access is framed around the physical materials with the CBD settling on a contract model that enables the party seeking access to negotiate a mutual agreement with the holder of the resources and share any benefits agreed between the parties from their uses of those physical materials. The CBD expressly provides for mutually agreed terms (Art 15.4), prior informed consent (Art 15.5) and "legislative, administrative or policy measures, as appropriate" to share "the results of research and development" and "benefits arising from the commercial and other utilization of genetic resources" (Art 15.7) and these are embedded in the contract instrument. The Nagoya Protocol essentially operationalizes the CBD's contract model providing the machinery provisions to enable access, such as focal points for information (Art 13.1) and authorities to issue permits that satisfy requirements of mutually agreed terms, prior informed consent and benefit sharing (Art 13.2). To engender confidence and trust in the arrangements there are also provisions for checkpoints to monitor and enhance transparency of uses (Art 17.1) and certificates of compliance (Arts 17.2-17.4). Similarly, the Plant Treaty and PIP Framework both adopt the contract model in the form of SMTAs dealing with the physical materials (Plant Treaty: Arts 12.4 and 15.1; PIP Framework: Art 5.4) and make provision for monitoring and transparency of uses (Plant Treaty: Arts 19.3(a) and 21; PIP Framework: Art 5.3). As confirmation of this physicality the PIP Framework distinguishes between "PIP biological materials" (Art 5.1.3) and GSD (Art 5.2.1) and deals separately with GSD (Art 5.2). 
As the article also demonstrates, each of the CBD, Nagoya Protocol, Plant Treaty and the PIP Framework provide for mechanisms to share and disclose information (CBD: Art 18.3 (CHM); Nagoya Protocol: Art 14.1 (Access and Benefit Sharing Clearing House); Plant Treaty: Art 17 (GLIS); PIP Framework: Art 5.2.2 (public domain and public access databases)). The conflict then is between information that is included as part of the ABS arrangements dealing with the physical materials - essentially treating the information as a resource derivative in the ABS transaction - and the broader obligations to share and disclose non-confidential or nontrade secret information. As the engagement of the governing bodies of the CBD, Nagoya Protocol, Plant Treaty and the PIP Framework have accepted, ABS arrangements are undermined if the information derived from the accessed materials are shared and disclosed outside the ABS transaction. This poses significant challenges, however, both to establishing mechanisms to monitor and trace the uses of information derived from the materials so that benefits can be extracted and the central tenet of modern research science that information be disclosed and shared to promote more and better science.

Monitoring and tracing the uses of information derived from DNA, RNA and proteins by monitoring and tracing sequence data has been shown to be problematic. A study using informatics techniques interrogating patent applications for evidence of the use of plant genetic resources derived from plants covered under the Plant Treaty demonstrated that monitoring and tracing is possible (see Oldham and Hall, 2013). The study used text mining to interrogate patent databases for key data, such as varieties, accession codes and for standardized variety denomination names (Oldham and Hall, 2013, p. 208). The study concluded that the informatics techniques were feasible when refined and could identify plant genetic resources in commercial research and development (Oldham and Hall, 2013, p. 208). The findings were, however, mired by "large-scale problems with noise" and being unable to "establish a clear linkage between plant germplasm in public collections and patent data" (Oldham and Hall, 2013, p. 208). The study essentially shows that informatics techniques can assist but that further detailed scrutiny is also required to determine the particular contributions such that using sequence data for monitoring and tracing is very complicated (PIP TEWG, 2014, pp. 11-12). While monitoring and tracing is appealing, the technical difficulties are considerable, as the patent database example using well 
characterized information in a form and context that was readily identifiable clearly demonstrates. Where the information is recorded in different formats and contexts, and where it has been fragmented for different uses, monitoring and tracing becomes increasingly problematic. In short, monitoring and tracing information is a theoretical possibility (see also GCD Monitoring, 2016), but in practice is unlikely to be a realistic method for extracting benefits. The considerable work so far under the PIP Framework and the preliminary scoping study under the CBD and Nagoya Protocol addressing monitoring and tracing have been unable to identify a clearly suitable method or mechanism (see A70/17, 2017, Annex (pp. 5354); UNEP/CBD/DSI/AHTEG/2018/1/3, 2018, [240]-[247]).

Perhaps the more significant challenge, however, comes from the central tenet of modern research science that information be disclosed and shared. This has been important both to verify and confirm the science concepts and to build on those concepts so that others can further develop those concepts, so building "by standing on the shoulder of giants" (Turnbull, p. 416). The example of the attempts over time to confine the disclosure and sharing of DNA, RNA and protein sequences has proven impossible (see Lawson and Rourke, 2016). The modern research science tenet has effectively demanded that DNA, RNA and protein sequences be made available and accessible. This tenet is reflected more broadly in the CBD, Nagoya Protocol, Plant Treaty and the PIP Framework mechanisms to share and disclose information (CBD: Art 18.3; Nagoya Protocol: Art 14.1; Plant Treaty: Art 17; PIP Framework: Art 5.2.2). Importantly, however, a closer assessment of the open access to DNA, RNA and protein sequences does show that modern research science is amenable to some restrictions, with evolving practices in sharing DNA, RNA and protein sequences expressly accepting restrictions where credit and attribution are sought by scientists or privacy protections are necessary (see Lawson and Rourke, 2016, 108-111). In other words, some regulation is acceptable, the question remains not that there be regulation but what type of regulation best suits the valuing of information? The challenge here is to maintain the legal obligations as the information passes between users making enforcement (including monitoring and tracing) difficult because the exchanges are often without legal obligations or the entity accessing the information may be hard or impossible to identify. These problems are further exacerbated by the fragmentation of information into networks of different users and using the information for different purposes and in different contexts. The possible solutions here, 
like those for monitoring and tracing, are theoretically possible but practically unlikely. The work so far under the PIP Framework and the preliminary scoping study under the CBD and Nagoya Protocol have been unable to identify a clearly suitable method or mechanism (see A70/17, 2017, Annex (p. 16); UNEP/CBD/DSI/AHTEG/2018/1/3, 2018, [150]-[162]).

While open and accessible information may be desirable for modern research science as it is presently structured, the practice in effect subsidizes some users in the value chain at the expense of others. Put another way, by excluding the providers of materials from which valuable information can be derived in sharing in the (potential) value though the $A B S$ transaction, those providers are in effect subsidizing the downstream beneficiaries that are able to capture more of the value that would otherwise be shared with the provider. The current focus of the CBD, Nagoya Protocol, Plant Treaty and the PIP Framework governing bodies is genetic information. The problem, however, is much broader and applies wherever the physical material is substitutable by information. This is likely to become increasingly problematic as the potential to derive information from physical materials improves and the capacity to apply analytic tools and techniques to process patterns, correlations and interactions from the available information delivers more usable and valuable information. As the stakeholders in the CBD, Nagoya Protocol, Plant Treaty and the PIP Framework increasingly engage with "digital sequence information" and GSD separate to the physical material, it is timely to devise practical solutions to overcome the multiple approaches to information sharing obligations.

A possible solution is to re-evaluate the contract model for the ABS transaction on which each of the CBD, Nagoya Protocol, Plant Treaty and the PIP Framework are based (see CBD: Art 15; Nagoya Protocol: Art 6; Plant Treaty: Arts 12.4 and 15.1; PIP Framework: Art 5.4). The options are essentially whether to maintain the contract ideal that the parties to the ABS transaction are best placed to determine the likely values of information and will include that in the conclusion of mutually agreeable terms for the exchange, or whether they externalize that cost in a separate arrangement that is paid separately from the ABS transaction, such as a charge, levy or tax. This will also require a re-evaluation of the mechanisms to share and disclose information as if this was a separate endeavour to the ABS transaction (CBD: Art 18.3; Nagoya Protocol: Art 14.1; Plant Treaty: Art 17; PIP Framework: Art 5.2.2). In effect, this re- 
valuation accepts that information is a derivative of the physical materials and, as such, it is a part of the ABS transaction. It will be important, however, to recognize that not all forms of information have the same values and that treating all information as of the same high value will impose unnecessary costs and a further administrative burdens on ABS transactions.

If the solution is to include information as a part of the ABS transaction, then the differing values of information might be determined according to a framework (matrix) of values reflecting the worth of particular kinds of information. Figure 1 merely illustrates the kinds of considerations that might be taken into account when considering the different values of information and the kinds of triggers that might apply to engage benefit sharing. This framework (matrix) is essentially a risk approach to valuing information and avoids attributing high value to all information when only some information is actually valuable. The key to this framework (matrix) is the assignment of the type of information and the assessment about whether the information is actually valuable. Clearly information that is subjected to trade secrecy or confidentiality is highly valued and the CBD, Nagoya Protocol and Plant Treaty already provide for its protection (CBD: Art 16.2; Nagoya Protocol, Art 4.4; Plant Treaty, Preamble). The details of such frameworks (matrices) will require refinement, and might be a suitable outcome in the form of voluntary guidelines under the existing CBD, Nagoya Protocol, Plant Treaty and the PIP Framework, like the CBD's Bonn Guidelines on Access to Genetic Resources and Fair and Equitable Sharing of the Benefits Arising out of their Utilisation (UNEP/CBD/COP/6/20, [342] and Annex I (Decision VI/24A, pp 253-269 (Bonn Guidelines)) were for the form and content of ABS contracts.

If the solution is to externalize the costs in a separate arrangement that is paid separately from the ABS transaction, this might include a charge, levy or tax. Various countries and private sector organisations are already making voluntary contributions to the Plant Treaty's Benefit Sharing Fund (Art 18.4(f); see IT/GB-7/17/Report, 2017, [6] and [28] and Appendix A.3 ([23] and [24]); Plant Treaty Secretariat, 2017). Meanwhile Norway has committed to an in perpetuity payment of 0.1 per cent of seed sales in Norway (IT/ACFS-6/10/Report, 2010, [7]). Under the PIP Framework there are subscription payments where influenza vaccine, diagnostic and pharmaceutical manufacturers using the WHO's system make a payment (called a 'Partnership Contribution') (Art 6.14.3). These payments and subscription are 
essentially already a charge, tax or levy. This charge, tax or levy might be paid either by those accessing the resources, as is the case under the PIP Framework, or as an impost on contracting parties, the Norway seed sales tax providing an illustrative mechanism. The great advantage of externalizing the costs in a separate arrangement is to release the ABS transaction of the high costs of negotiating the value of information in each transaction and the central tenet of modern research science that information be disclosed and shared.

\section{Conclusion}

The article shows that a closed domain for information under the ABS schemes where information is a resource derivative that is part of the ABS transaction will directly conflict with the open domain required by the information sharing obligations. The enclosure here is mediated through shifting the handling of information into the ABS domain and away from the existing ideals of open disclosure and exchange of information about genetic resources. The innovative approach postulated in this article is a risk framework (matrix) for valuing information as a part of the ABS transaction, or a charge, tax or levy to externalize the costs so that information remains available to be disclosed and exchanged promoting more and better science and research. It is timely for more research about the merits of these proposals before the international forums lock in a particular approach to information sharing that may inhibit the fair and equitable sharing of genetic resource information and the progress of modern science. 
Figure 1: Framework (matrix) of values reflecting the worth of particular kinds of information.

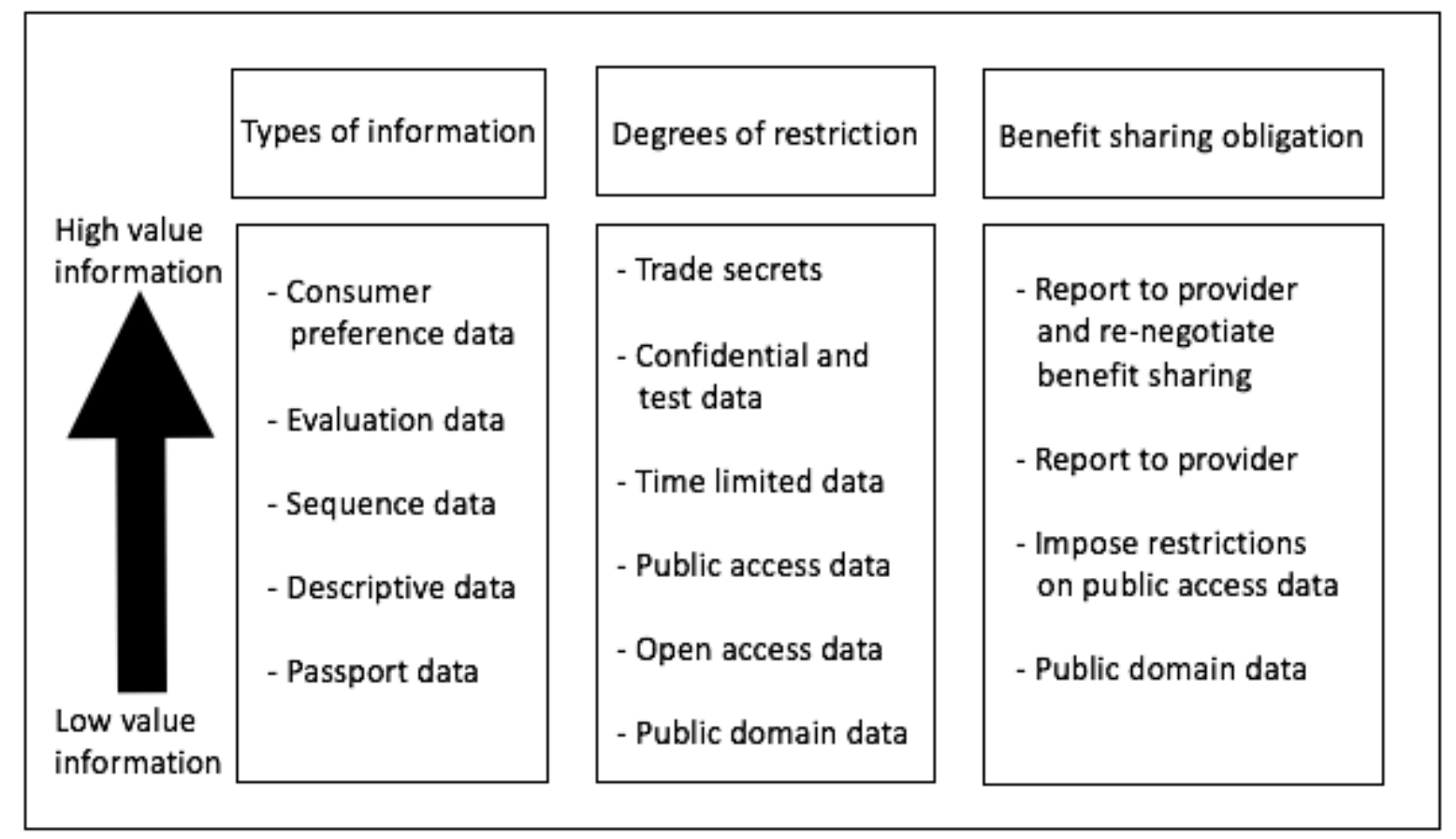

\section{About the authors}

Dr. Charles Lawson is a member of the Australian Centre for Intellectual Property in Agriculture at the Griffith Law School, Griffith University, Australia.

Dr. Fran Humphries is a Research Fellow at the Law Futures Centre and member of the Australian Centre for Intellectual Property in Agriculture at the Griffith Law School, Griffith University, Australia.

Michelle Rourke is a Scientific Research Officer at the Australian Defence Force Malaria and Infectious Disease Institute in Brisbane, Australia and a PhD candidate at the Griffith Law School, Griffith University, Australia. The opinions expressed here are those of the author and do not reflect any view or position of the Australian Defence Force Malaria and Infectious Disease Institute, the Australian Defence Force or the Commonwealth of Australia. 


\section{Endnotes}

1. There is a distinction between genetic resources, biological resources and derivatives. Usefully the CBD defines "biological resources" as including "genetic resources, organisms or parts thereof, populations, or any other biotic component of ecosystems with actual or potential use or value for humanity" (art 2), "genetic resources" as "genetic material of actual or potential value" where "genetic material" means "any material of plant, animal, microbial or other origin containing functional units of heredity" (art 2), and the Nagoya Protocol defines "derivative" to mean "a naturally occurring biochemical compound resulting from the genetic expression or metabolism of biological or genetic resources, even if it does not contain functional units of heredity" (art 2).

2. Some countries have adopted the broader term "biological resources" to prevent the loophole where access to biological resources for consumptive uses can lead to the use of their genetic or chemical composition without prior informed consent or mutually agreed terms: see Morgera et al., 2016, p. 72.

3. The Commission on Genetic Resources for Food and Agriculture was careful of its use of terminology taking this terminology from the Conference of the Parties Decision XIII/16: see CGRFA-16/17/Report, 2017, [86], footnote 50; UNEP/CBD/COP/13/25, 2016, [321] and Decision XIII/16 (pp. 124-125).

4. The Governing Body was careful of its use of terminology taking this terminology from the Conference of the Parties Decision XIII/16: see IT/GB-7/17/Report, 2017, [43] and Appendix A.13 (Resolution 13/2017, p. 60, footnote 48); UNEP/CBD/COP/13/25, 2016, [321] and Decision XIII/16 (pp. 124-125).

5. Global Initiative on Sharing Avian Influenza Data (GISAID) available at <http://www.gisaid.org> (accessed 27 March 2018).

6. "GenBank" at the National Center for Biotechnology Information available at <http://www.ncbi.nlm.nih.gov/genbank> (accessed 27 March 2018).

7. CBD "Clearing House Mechanism" <http://www.chm-cbd.net> (accessed 27 March 2018). 


\section{Bibliography}

A66/17 (2013) World Health Organisation, Pandemic Influenza Preparedness: Sharing of Influenza Viruses and Access to Vaccines and Other Benefits: Pandemic Influenza Preparedness Framework 2013 Biennial Report, WHO: Geneva.

A67/36 (2014) World Health Organisation, Pandemic Influenza Preparedness: Sharing of Influenza Viruses and Access to Vaccines and Other Benefits: Pandemic Influenza Preparedness Framework, WHO: Geneva.

A69/22 Add.1 (2016) World Health Organisation, Pandemic Influenza Preparedness: Sharing of Influenza Viruses and Access to Vaccines and Other Benefits: Report of the Special Session of the Pandemic Influenza Preparedness Framework Advisory Group, WHO: Geneva.

A69/22 Add.2 (2016) World Health Organisation, Pandemic Influenza Preparedness: Sharing of Influenza Viruses and Access to Vaccines and Other Benefits: Report by the DirectorGeneral, WHO: Geneva.

A70/17 (2017) World Health Organisation, Review of the Pandemic Influenza Preparedness Framework, WHO: Geneva.

Access to Biological Resources and Benefit Sharing Act 2017 (Mal)

Bagley, Margo and Arti Rai, The Nagoya Protocol and Synthetic Biology Research: A Look at the Potential Impacts (Wilson Centre, 2014).

CBD (1992) Convention on Biological Diversity 1760 U.N.T.S. 79, 31 I.L.M. 818.

CGRFA-16/17/Report (2017) Commission on Genetic Resources for Food and Agriculture, Sixteenth Regular Session of the Commission on Genetic Resources for Food and Agriculture, CGRFA: Rome.

GSD Monitoring (2016) World Health Organization, Best Process to Handle Genetic Sequence Data from Influenza Viruses with Human Pandemic Potential (IVPP GSD) under the PIP Framework: Options to Monitor the Use of Genetic Sequence Data from Influenza Viruses with Human Pandemic Potential (IVPP GSD) in End-products available at <http://www.who.int/influenza/pip/advisory_group/gsdoptionspaper_revised.pdf> (accessed 27 March 2018).

EB144/CONF./10 (2019) Executive Board, Pandemic Influenza Preparedness Framework for the Sharing of Influenza Viruses and Access to Vaccines and Other Benefits, WHO: Geneva. 
IT/ACFS-6/10/Report (2010) Ad Hoc Advisory Committee on the Funding Strategy, Sixth Meeting of the Ad Hoc Advisory Committee on the Funding Strategy, FAO: Rome.

IT/GB-1/06/Report (2006) Governing Body of the International Treaty on Plant Genetic Resources for Food and Agriculture, First Session of the Governing Body of the International Treaty on Plant Genetic Resources for Food and Agriculture, FAO: Rome.

IT/GB-5/13/4 (2013) Governing Body of the International Treaty on Plant Genetic Resources for Food and Agriculture, Report of the Secretary, FAO: Rome.

IT/GB-5/13/Report (2013) Governing Body of the International Treaty on Plant Genetic Resources for Food and Agriculture, Fifth Session of the Governing Body of the International Treaty on Plant Genetic Resources for Food and Agriculture, FAO: Rome.

IT/GB-6/15/Report (2015) Governing Body of the International Treaty on Plant Genetic Resources for Food and Agriculture, Sixth Session of the Governing Body of the International Treaty on Plant Genetic Resources for Food and Agriculture, FAO: Rome.

IT/GB-7/17/Report (2017) Governing Body of the International Treaty on Plant Genetic Resources for Food and Agriculture, Seventh Session of the Governing Body of the International Treaty on Plant Genetic Resources for Food and Agriculture, FAO: Rome.

IT/GB7/SAC-1/16/BSP 10 (2016) First Meeting of the Scientific Advisory Committee on the Global Information System of Article 17 of the Treaty, The Global Information System and Genomic Information: Transparency of Rights and Obligations, FAO: Rome.

IT/SAC-GLIS-3/18/Report (2018) Third Meeting of the Scientific Advisory Committee on the Global Information System of Article 17 of the Treaty, Report, FAO: Rome.

Laird, Sarah and Rachel Wynberg (2016) "Locating Responsible Research and Innovation Within Access and Benefit Sharing Spaces of the Convention on Biological Diversity: The Challenge of Emerging Technologies", Nanoethics 10: 189-200.

Lawson, Charles, Regulating Genetic Resources: Access and Benefit-sharing in International Law (Edward Elgar, 2012).

Lawson, Charles and Michelle Rourke (2016) "Open Access DNA, RNA and Amino Acid Sequences: The Consequences and Solutions for the International Regulation of Access and Benefit Sharing", Journal of Law and Medicine 24(1): 96-118.

Malbon, Justin, Charles Lawson and Mark Davison, The WTO Agreement on Trade-Related Aspects of Intellectual Property Rights: A Commentary (Edward Elgar, 2014). 
Morgera, Elisa, Elsa Tsioumani and Matthias Buck, Unravelling the Nagoya Protocol (Brill, 2016).

Muller, Manuel Ruiz (2018) "Reshaping the International Access to Genetic Resources and Benefit Sharing Process?: Overcoming Resistance to Change and Correction" in Charles Lawson and Kamalesh Adhikari (eds.), Biodiversity, Genetic Resources and Intellectual Property: Developments in Access and Benefit Sharing (Routledge, 2018) pp. 208-219.

Nagoya Protocol (2010) UNEP/CBD/COP/10/27, Conference of the Parties to the Convention on Biological Diversity, Report of the Tenth Meeting of the Conference of the Parties to the Convention on Biological Diversity, [103] and Annex (Decision X/1, Annex 1, pp. 89-109), CBD: Montreal.

Noyce, Ryan, Seth Lederman and David Evans, "Construction of an Infectious Horsepox Virus Vaccine from Chemically Synthesized DNA Fragments" (2018) PLoS One 13(1): e0188453.

Oldham Paul and Stephen Hall, "Intellectual Property, Informatics and Plant Genetic Resources" in Nina Moeller and Clive Stannard (eds.), Identifying Benefit Flows: Studies on the Potential Monetary and Nonmonetary Benefits Arising from the International Treaty on Plant Genetic Resources for Food and Agriculture (Food and Agriculture Organization of the United Nations, 2013) pp. 162-223.

PIP FAG DG (2014) Pandemic Influenza Preparedness Framework Advisory Group, Report to the Director-General available at <http://www.who.int/influenza/pip/pip_ag_oct2014_meetingreport_final_7nov201 4.pdf?ua=1> (accessed 27 March 2018).

PIP FAG TWG (2014) Pandemic Influenza Preparedness Framework Advisory Group, Technical Working Group (TWG) on the Sharing of Influenza Genetic Sequence Data: Terms of Reference available at <http://www.who.int/influenza/pip/advisory_group/twg_tors.pdf?ua=1> (accessed 27 March 2018).

PIP FAG TWG GSD (2015) Pandemic Influenza Preparedness Framework Advisory Group Meeting of the Technical Working Group on the Sharing of Influenza Genetic Sequence Data, Meeting Report available at 
<http://www.who.int/influenza/pip/advisory_group/twg_sept2015meeting.pdf?ua= 1> (accessed 27 March 2018).

PIP FAG TWG GSD (2016) Pandemic Influenza Preparedness (PIP) Framework Advisory Group Technical Working Group (TWG) on the Sharing of Influenza Genetic Sequence Data, Optimal Characteristics of an Influenza Genetic Sequence Data Sharing System under the PIP $\quad$ Framework available at <http://www.who.int/influenza/pip/advisory_group/twg_doc.pdf?ua=1> (accessed 27 March 2018).

PIP Framework (2011) WHA64.5, World Health Organisation, Pandemic Influenza Preparedness: Sharing of Influenza Viruses and Access to Vaccines and Other Benefits, Sixty-Fourth World Health Assembly, WHO: Geneva.

PIP TEWG (2014) Pandemic Influenza Preparedness Framework Advisory Group, Pandemic Influenza Preparedness Framework Advisory Group Technical Expert Working Group (TEWG) on Genetic Sequence Data: Final Report to the PIP Advisory Group available at <http://www.who.int/influenza/pip/advisory_group/PIP_AG_Rev_Final_TEWG_Repo rt_10_Oct_2014.pdf> (accessed 27 March 2018).

Plant Treaty (2001) International Treaty for Plant Genetic Resources for Food and Agriculture 2400 U.N.T.S. 303.

Plant Treaty Secretariat (2017) Secretariat of the International Treaty on Plant Genetic Resources for Food and Agriculture, French Seed Sector Announces Annual Contribution to Benefit Sharing Fund (2017) available at <http://www.fao.org/planttreaty/news/news-detail/en/c/1054532> (accessed 27 March 2018).

Roberts, Leslie, “Chemical Prospecting: Hope for Vanishing Ecosystem?" (1992) 256 Science 1142.

Schiele, Simone, Deborah Scott, Dina Abdelhakim et al. (2015) Possible Gaps and Overlaps with the Applicable Provisions of the Convention, its Protocols and Other Relevant Agreements Related to Components, Organisms and Products Resulting from Synthetic Biology Techniques: Synthetic Biology Part II, Technical Series No 82 (CBD Secretariat, 2015).

Svarstad, Hanne, "National Sovereignty and Genetic Resources" in in Vincent Sánchez and Calestous Juma (eds.), Biodiplomacy: Genetic Resources and International Relations (ACTS Press, 1994) pp. 45-65. 
TRIPS (1994) Trade-Related Aspects of Intellectual Property Rights as Marrakesh Agreement Establishing the World Trade Organization (1994) Annex 1C, 1869 U.N.T.S. 299, 33 I.L.M. 1197.

Turnbull H (Editor), The Correspondence of Isaac Newton, Volume 1 (Cambridge University Press, 1959).

UNEP/CBD/COP/2/13 (1995) Conference of the Parties to the Convention on Biological Diversity, Access to Genetic Resources and Benefit Sharing: Legislation, Administrative and Policy Information, CBD: Montreal.

UNEP/CBD/COP/2/19 (1995) Conference of the Parties to the Convention on Biological Diversity, Report of the Second Meeting of the Conference of the Parties to the Convention on Biological Diversity, CBD: Montreal.

UNEP/CBD/COP/3/20 (1996) Conference of the Parties to the Convention on Biological Diversity, Access to Genetic Resources, CBD: Montreal.

UNEP/CBD/COP/4/27 (1998) Conference of the Parties to the Convention on Biological Diversity, Report of the Fourth Meeting of the Conference of the Parties to the Convention on Biological Diversity, CBD: Montreal.

UNEP/CBD/COP/6/20 (2002) Conference of the Parties to the Convention on Biological Diversity, Report of the Sixth Meeting of the Conference of the Parties to the Convention on Biological Diversity, CBD: Montreal.

UNEP/CBD/COP/12/29 (2013) Conference of the Parties to the Convention on Biological Diversity, Report of the Twelfth Meeting of the Conference of the Parties to the Convention on Biological Diversity, CBD: Montreal.

UNEP/CBD/COP/13/5 (2015) Conference of the Parties to the Convention on Biological Diversity, Report of the Subsidiary Body on Scientific, Technical and Technological Advice on its Twentieth Meeting, CBD: Montreal.

UNEP/CBD/COP/13/25 (2016) Conference of the Parties to the Convention on Biological Diversity, Report of the Conference of the Parties to the Convention on Biological Diversity on its Thirteenth Meeting, CBD: Montreal.

UNEP/CBD/COP/DEC/14/20 (2018) Conference of the Parties to the Convention on Biological Diversity, Decision Adopted by the Conference of the Parties to the Convention on Biological Diversity - 14/20. Digital sequence information on genetic resources, CBD: Montreal. 
UNEP/CBD/DSI/AHTEG/2018/1/3 (2018) Ad Hoc Technical Expert Group on Digital Sequence Information on Genetic Resources, Fact-Finding and Scoping Study on Digital Sequence Information on Genetic Resources in the Context of the Convention on Biological Diversity and the Nagoya Protocol, CBD: Montreal.

UNEP/CBD/ICNP/3/6 (2014) Open-Ended Ad Hoc Intergovernmental Committee for the Nagoya Protocol on Access to Genetic Resources and the Fair and Equitable Sharing of Benefits Arising from their Utilization, Report on Progress in the Implementation of the Pilot Phase of the Access and Benefit Sharing Clearing-House, CBD: Montreal.

UNEP/CBD/NP/MOP/2/13 (2016) Conference of the Parties to the Convention on Biological Diversity Serving as the Meeting of the Parties to the Nagoya Protocol on Access to Genetic Resources and the Fair and Equitable Sharing of the Benefits Arising from their Utilization, Report of the Conference of the Parties to the Convention on Biological Diversity Serving as the Meeting of the Parties to the Nagoya Protocol on Access to Genetic Resources and the Fair and Equitable Sharing of the Benefits Arising from their Utilization on its Second Meeting, CBD: Montreal.

UNEP/CBD/SYNBIO/AHTEG/2015/1/3 (2015) Ad Hoc Technical Expert Group on Synthetic Biology, Report of the Ad Hoc Technical Expert Group on Synthetic Biology, CBD: Montreal.

UNEP/CBD/WG-ABS/7/2 (2008) Ad Hoc Open-Ended Working Group on Access and Benefit Sharing, Report of the Meeting of the Group of Legal and Technical Experts on Concepts, Terms, Working Definitions and Sectoral Approaches, CBD: Montreal.

UNEP/CBD/WG-ABS/9/INF/1 (2010) Ad Hoc Open-Ended Working Group on Access and Benefit Sharing, The Concept of "Genetic Resources" in the Convention on Biological Diversity and How It Relates to a Functional International Regime on Access and Benefit Sharing, CBD: Montreal.

UNEP/CBD/WGRI/5/3/Add.2 (2014) Ad Hoc Open-Ended Working Group on Review of Implementation of the Convention, Progress Report on the Clearing House Mechanism, CBD: Montreal.

Welch, Eric, Margo Bagley, Todd Kuiken and Sélim Louafi, Potential implications of new synthetic biology and genomic research trajectories on the International Treaty for Plant Genetic Resources for Food and Agriculture - Scoping Report (Food and Agriculture Organization of the United Nations, 2017). 
Wynberg, Rachel and Sarah Laird, "Bioprospecting, Access and Benefit Sharing: Revisiting the 'Grand Bargain'” in Rachel Wynberg, Doris Schroeder and Roger Chennells (eds.), Indigenous Peoples, Consent and Benefit Sharing: Lessons from the San-Hoodia Case (Springer, 2009) pp. 69-86. 